W hat's your use-by-date? There has been controversy about whether clinicians get better or worse with age. The Notebook in this issue discusses the interpretation of a recent systematic review on the topic. It makes essential reading. The Practice Corner in this issue looks at a question that has troubled me since medical school: what is the risk of re-seizure after a first seizure? Prognosis is generally less well researched and synthesised than therapeutics, so this article gives some useful tips and tactics. However, it would be lovely to see a Cochrane equivalent for natural history and prognosis, as this is fundamental to informed patient choices. We also continue our series on short exposés of tricky statistical issues: this time the "weighted event rates" often used in meta-analyses. If you have a particular statistical issue you'd like to see covered, please let us know. We would also welcome short items on EBM related issues, such as teaching tips, how you got interested in EBM, or relevant quotes.

Happy reading,

PAUL GLASZIOU, MBBS, PhD University of Oxford Oxford, UK

\title{
Does clinical experience make up for failure to keep up to date?
}

Y ou have just moved to a new town to open up your internal medicine practice, and now must find a doctor for your own young family. Two doctors are accepting new patients:

Jane completed residency 2 years ago, and scored in the top $2 \%$ of her class on the certification exams.

Susan completed residency 10 years ago, and scored in the top $25 \%$ of her class on the certification exams.

Who do you choose?

When this question has been posed to large clinical audiences over the past few years, only half a dozen people ever choose Jane over Susan. Why? Most people would accept that not only did Susan start off worse in formal knowledge, but also that it is very likely that her knowledge and application of current recommended care is likely to have fallen off further, as Choudhry et al have shown in a recent systematic review. ${ }^{1}$ Why, then, do audiences so unanimously choose the more experienced practitioner?

Perhaps for just that reason-experience. The practice of medicine, like many other areas of human endeavour, requires considerable "hands on" experience to achieve mastery. Most physicians, when asked, indicate that they did not really feel competent for several years after they entered practice, which tallies with estimates from other domains that suggest 10 years or 10000 hours are required to become a virtuoso. ${ }^{2}$ But what is gained from experience?

Unfortunately, if you believe the conclusions of Choudhry et al, the answer is that nothing is gained and much is lost. They claim to have identified several studies where increasing years in practice are associated with increased mortality. But on closer scrutiny, the differences, when present, are small. The paper states that, in the best study of outcome, ${ }^{3}$ every year since graduation resulted in an increase of $0.5 \%$ mortality in the management of post-myocardial infarction (MI) patients. However, this was an increase in relative risk so that each year of practice equated to an absolute increase of mortality of only about $0.05 \%$, on a baseline mortality rate post-MI of about $10 \%$. After 20 years of practice, the mortality would be projected to rise to $12 \%$. A second study by the same authors ${ }^{4}$ showed a non-significant increase corresponding to about $0.02 \%$ mortality per year, or an absolute increase in risk of $0.4 \%$ after 20 years. Since treatment of MI is an area of medicine where swift advances in treatment are the norm, this figure might well be regarded as an upper limit, and indeed closer examination of the other studies of mortality cited by Choudhry et al show small or absent effects.

These findings reveal an intriguing paradox. Physicians in practice tend not to keep up on either knowledge tests or adherence to practice guidelines, but this seems to have minimal impact, if any, on patient outcomes. It could, of course, be the case that practice guidelines do not make that much difference to patient outcomes, and indeed there are few studies showing a benefit of guidelines. ${ }^{5}$ However, it is not the case that outcomes are simply insensitive to provider differences, because, in the studies by Norcini et $\mathrm{al}^{3}{ }^{3}$ subspecialisation resulted in absolute mortality differences of about $2.5 \%$, and success on a certification examination resulted in an absolute mortality difference of about $2 \%$. So we are left with the conclusion that the outcome measures do not seem to decline with age commensurate with the drop in knowledge or guideline compliance. Does that mean that there is something acquired from experience that is able to compensate for a fairly large decline in knowledge and therapeutic approach?

Some indication of this compensatory mechanism can be gleaned from studies of expertise in other domains. The most studied area of expertise is chess, which has been 
systematically explored for over 50 years. It has been shown that chess expertise is due, in large part, to thousands of hours of deliberate practice, taking 10 to 20 years. $^{2}$ The consequence is that chess performance shows a curvilinear relation to age, peaking at about age 35 while expertise is slowly acquired, and declining slowly thereafter.

Expert chess play consists, in large part, of matching the current play to some learned moves in memory-what one might call pattern recognition. Not surprisingly, since this is the essential nature of the skill, chess expertise is best observed in speeded play ${ }^{6}$ where the player must rapidly select the best move, and where the ability to recognise patterns of play is a decided asset. Studies in other areas of expertise have also shown that experts actually do better under speeded conditions; expert golfers have more accurate putts when told to be rapid than when told to be accurate. ${ }^{7}$

Some similar observations exist in medicine. Expert dermatologists, when they are right, are quick-an average of 8 seconds. When they are wrong, it takes them about 12 seconds, and when they are unsure, they will ponder for 28 seconds on average. ${ }^{8}$ Hobus et al have shown that when clinicians are provided with minimal information, the correlation between diagnostic accuracy and experience is +0.68 .

Although these findings suggest that experience enables practitioners to make decisions rapidly, it remains unclear how this skill relates to experience. We have pursued a line of inquiry based on the assertion that expert clinicians frequently arrive at a diagnosis by mentally comparing the presenting situation to a specific previous case. ${ }^{10-12}$ The process occurs without conscious reflection, analogous to the way we recognise a friend on the street. ${ }^{12}$ Thus, it is reasonable to presume that a major component of medical expertise that is learned during the course of many years of practice is the accumulation of a vast mental storehouse of clinical cases, on which experts draw repeatedly in arriving at a diagnosis.

Cast in this light, the real perplexity in the review by Choudhry et al is the lack of a positive relation between experience and clinical outcomes. Although the clinician becomes more and more accomplished at diagnosis based on pattern recognition processes with increasing experience, it may be the case that the benefits of the strategy come at the cost of reduced flexibility. Hashem et al ${ }^{13}$ have presented data showing that specialists have a tendency to cognitively "pull" cases toward the domains with which they have most experience.

Confirmation of this possibility comes from studies of the Physician Review and Enhancement Program (PREP) in Ontario, Canada, using a battery of tests of physician performance. Overall, they too found a negative association between age and expertise. ${ }^{14}$ Systematic consideration of the causes of poor performance in the older physicians, however, suggests that premature closure (ie, excessive reliance on one's early impressions of a case) is a major source of difficulty. ${ }^{15}$ In other words, more experienced physicians seem more likely to accurately diagnose using pattern recognition, but as a result of increased reliance on this strategy, they are also less likely to give due consideration to competing diagnoses. ${ }^{16}$

The discussion above relates primarily to diagnostic expertise. But by and large, the performance measures in the review by Choudhry et al reflect either surgical skill or management strategies, in circumstances where the diagnosis is a given. However, a recent study sheds some light on the relation between experience and management. Schuwirth et $a l^{17}$ assessed rheumatologists with 2 kinds of clinical problems: A computer-based test consisting of 55 written management cases focusing on 1-4 essential decisions, and a series of 8 incognito standardised patients who visited their offices and completed a performance checklist after the encounter. The standardised patient test had a strong negative correlation $(r=-0.50)$ with the total number of patients seen during the rheumatologist's professional life, again presumably because experts do not require as much information as novices, so their expertise is impaired by use of checklist scoring systems. ${ }^{18}$ In contrast, the computer test was positively correlated $(r=+0.58)$ with lifetime experience. Why the discrepancy with Choudhry et al's findings? Perhaps because responses were subjectively scored by other experts on a "case by case" basis, rather than compared with a detailed set of items like a practice guideline. Perversely, it may just be that expertise is evidenced as much in knowing when to depart from guidelines as knowing what the guidelines say. Indeed, a recent study ${ }^{19}$ of hospital clinicians indicated that consultants' approaches to drug treatment were more idiosyncratic than those of house officers, mainly because the consultants were more holistic and adapted the prescribing to the individual patient, whereas junior doctors used a more formulaic approach.

In summary, we have suggested 2 mechanisms to explain the paradox that apparently large declines in measures of knowledge and process of care do not translate into commensurate large differences in patient outcome. Firstly, adherence to prescribed practices of care may be, in some sense, optimal at a population level, but at an individual level, experienced physicians may deliberately and systematically depart from these guidelines to recognise individual patient needs. As a consequence, they may be penalised on measures based on adherence to prescribed regimens. Secondly, there is an accumulation of evidence that with experience, physicians rely more on pattern recognition strategies that can, to some degree, compensate for failure to keep up in formal knowledge, but can themselves lead to negative consequences. ${ }^{20}$

We are not suggesting that the findings of Choudhry et al should be lightly dismissed. They do indicate that physicians are not keeping up with current approaches to patient care. Current approaches to maintenance of competence that are dependent on self assessment of one's own knowledge/ abilities should be critically re-examined. It seems unlikely that admonitions to be more reflective or to identify weaknesses can overcome the negative trends identified.

Viewing experience as a double edged sword, as we have, creates the opportunity for more effective continuing education. Lectures and distribution of printed materials are not effective. ${ }^{21}$ Learning around specific cases in which individuals are challenged to apply the latest research evidence might be. ${ }^{22}$ Doing so in a context in which participants are required to respond to feedback is likely to provide further incremental benefit, especially if that feedback is derived from individuals with heterogeneous backgrounds and varied levels of expertise. In general, the position presented in this editorial leads us to advocate recognizing the unique strengths that experience provides while simultaneously developing and investigating continuing education strategies that re-ignite the analytic tendencies of individuals for whom medical practice has become excessively automated and routinised.

GEOFFREY R NORMAN, PHD KEVIN W EVA, PHD McMaster University, Hamilton, Ontario, Canada 
68

1 Choudhry NK, Fletcher RH, Soumerai SB. Systematic review: the relationship between clinical experience and quality of health care. Ann Intern Med 2005; 142:260-73.

2 Ericsson KA. Deliberate practice and the acquisition and maintenance of expert performance in medicine and related domains. Acad Med 2004;79:S70-81.

3 Norcini JJ, Kimball HR, Lipner RS. Certification and specialization: do they matter in the outcome of acute myocardial infarction? Acad Med 2000;75: 1193-8.

4 Norcini JJ, Lipner RS, Kimball HR. Certifying examination performance and patient outcomes following acute myocardial infarction. Med Educ 2002;36:853-9.

5 Straus $\mathrm{SE}$, Jones $\mathrm{G}$. What has evidence based medicine done for us? BMJ 2004:329:987-8.

6 Burns BD. The effects of speed on skilled chess performance. Psychol Sci 2004:15:442-7.

7 Beilock SL, Bertenthal BI, McCoy AM, et al. Haste does not always make waste: expertise, direction of attention, and speed versus accuracy in performing sensorimotor skills. Psychon Bull Rev 2004;11:373-9.

8 Norman GR, Rosenthal D, Brooks LR, et al. The development of expertise in dermatology. Arch Dermatol 1989;125:1063-8.

9 Hobus PP, Schmidt HG, Boshuizen HP, et al. Contextual factors in the activation of first diagnostic hypotheses: expert-novice differences. Med Educ 1987;21:471-6.

10 Allen SW, Norman GR, Brooks LR. Experimental studies of learning dermatologic diagnosis: the impact of examples. Teach Learn Med 1991;4:35-44.

11 Kulatunga-Moruzi C, Brooks LR, Norman GR. Coordination of analytic and similarity-based processing strategies and expertise in dermatological diagnosis. Teach Learn Med 2001;13:110-6.
12 Hatala R, Norman GR, Brooks LR. Influence of a single example upon subsequent electrocardiogram interpretation. Teach Learn Med 1999;11:110-7.

13 Hashem A, Chi MT, Friedman CP. Medical errors as a result of specialization. $J$ Biomed Inform 2003;36:61-9.

14 Norman GR, Davis DA, Lamb S, et al. Competency assessment of primary care physicians as part of a peer review program. JAMA 1993:270:1046-51.

15 Caulford PG, Lamb SB, Kaigas TB, et al. Physician incompetence: specific problems and predictors. Acad Med 1994;69:S16-8.

16 Eva KW. The aging physician: changes in cognitive processing and their impact on medical practice. Acad Med 2002;77:S1-6.

17 Schuwirth L, Gorter S, van de Heijde D, et al. The role of a computerized casebased testing procedure in practice performance assessment. Adv Health Sci Educ Theory Pract 2005; (in press).

18 Hodges B, Regehr G, McNaughton N, et al. OSCE checklists do not capture increasing levels of expertise. Acad Med 1999;74:1129-34.

19 Higgins MP, Tully MP. Hospital doctors and their schemas about appropriate prescribing. Med Educ 2005;39:184-93.

20 Eva KW. What every teacher needs to know about clinical reasoning. Med Educ 2005;39:98-106.

21 Grimshaw JM, Shirran L, Thomas R, et al. Changing provider behavior: an overview of systematic reviews of interventions. Med Care 2001;39:॥2-45.

22 Herbert CP, Wright JM, Maclure $M$, et al. Better Prescribing Project: a randomized controlled trial of the impact of case-based educational modules and personal prescribing feedback on prescribing for hypertension in primary care. Fam Pract 2004;21:575-81.

\section{Practice corner: will it happen again doctor? Prognosis after a first seizure}

\section{SCENARIO}

Susan attends with her mother for a consultation at the end of Monday morning surgery. On the previous Friday evening, 22 year old Susan was alone in the kitchen when her mother heard a clatter of kitchen utensils. She went through to find Susan unconscious on the floor in a pool of urine with her arms and legs shaking. This subsided after a couple of minutes leaving Susan drowsy and confused. Susan takes no regular medication and has had no serious illnesses in the past. There is no suspicion that alcohol or illicit drugs are involved. This is the first time that something like this has ever happened. She was seen in the local accident and emergency department and allowed home after being reassured that her blood tests, heart tracing, and clinical examination were all entirely normal. A neurological outpatient appointment is being organised, and Susan has been told that she mustn't drive.

\section{CLINICAL PREDICAMENT}

Both Susan and her mother are very worried that she's going to have another convulsion. We discuss some of the implications of her recent convulsion and the difficulty of adopting an essentially "wait and see" approach pending further neurological assessment. I'm reluctant to hazard a guess as to the likelihood of a recurrence. My clinical intuition is that the risk of recurrence is quite high (if pushed I'd estimate $70 \%$ over 12 months). A straw poll of 6 general practitioner (GP) colleagues over morning coffee produced a wider range of estimates ( 10 , $20,40,60$, and $70 \%)$. But I'm conscious that none of our estimates are based on extensive clinical experience. Primary care physicians only see around 1 new patient with a first seizure every couple of years. ${ }^{1}$

\section{SEARCHING CLINICAL QUERIES}

The PubMed Clinical Queries filters include a focused search for research relating to "prognosis." The search can set to be either broad (include a lot of irrelevant articles, but unlikely to miss many relevant ones) or narrow (fewer irrelevant articles, but also likely to miss a few relevant ones). I used the free text term first convulsion (figure 1), reasoning that a clinical researcher interested in prognosis would probably use the term convulsion (a clinical presentation) rather than epilepsy (implying a more certain diagnosis).

A narrow search yielded 260 references (table), which is far more than I've got time to scroll through (a broad search locates even more citations, 586). Selecting the alternative "systematic reviews" option reduces the number of references to 29. The seventh article is "an evidence based approach to the first unprovoked seizure." ${ }^{2}$ Unfortunately the PubMed abstract contains no prognostic data, but it does indicate that the article includes a 1 page critical appraisal of the topic (CAT) based on a published meta-analysis.

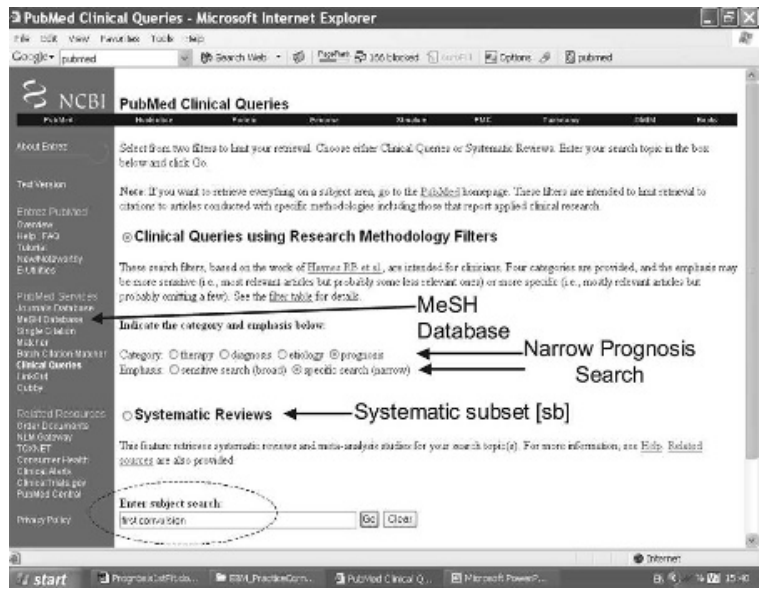

Figure 1. 


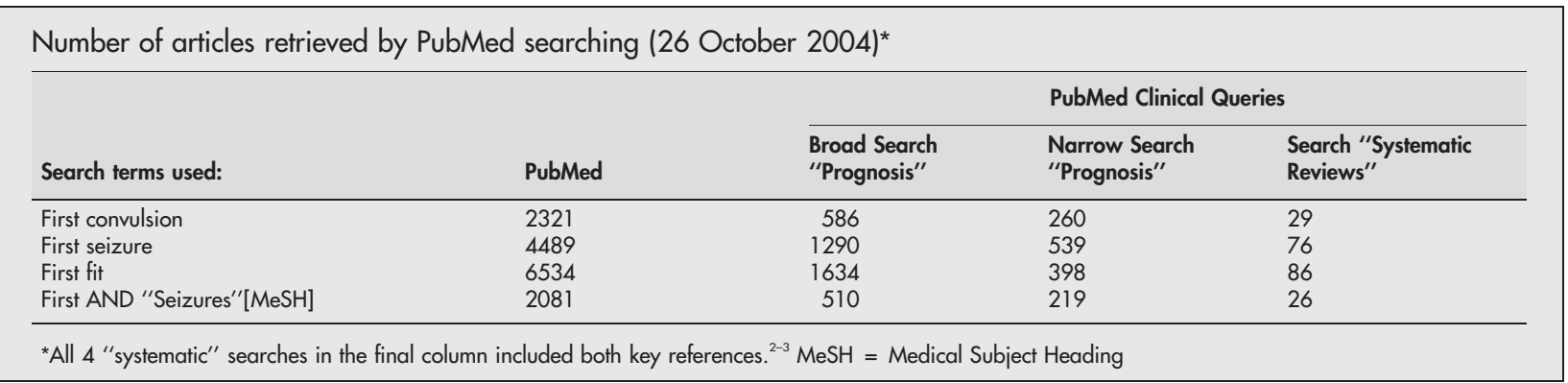

PubMed often has direct links to full text journals, but not this time. Google (www.google.com) is the quickest way that I know to check if a journal is available electronically (Google is so useful for evidence-based medicine [EBM] that I've installed the free toolbar on my web browser). Googling Canadian Journal Neurological Sciences rapidly locates their website, which is "celebrating 30 year of publishing" by providing free online access (www.cjns.org). The selected article $^{2}$ was actually written to illustrate the EBM approach and it critically appraises the published meta-analysis against explicit criteria. ${ }^{4}$

\section{PROGNOSIS AFTER FIRST SEIZURE}

Based on pooled results (of 1930 patients from 13 studies with $\geqslant 12$ months' follow up), the overall risk of seizure recurrence after a first unprovoked seizure is $42 \%$ (95\% CI $39 \%$ to $44 \%$ ) after 2 years' follow up. ${ }^{2}$ An increased risk of recurrence is associated with abnormal neurological findings (which Susan doesn't have) and an abnormal EEG. ${ }^{2}$ A quick look at the PubMed abstract for the original meta-analysis (also picked up on my search) adds little further information, other than generalised seizures having a lower recurrence risk than partial seizures. ${ }^{3}$ Although the meta-analysis was published rather a long time ago (1991), it was still the only systematic review available when the CAT was produced in September 2001. ${ }^{2}$ The journal Neurology is available online (Googling journal neurology immediately locates www.neurology.org), but unfortunately electronic full text is only available from January 1999.

\section{GOOGLING}

Google (www.google.com) is also very useful for quickly locating other important documents. For example, Googling fitness to drive immediately locates the DVLA (Driver Vehicle Licensing Agency) current medical standards for driving (www.dvla.gov.uk/at_a_glance/content.htm). The latest version (updated September 2004) indicates that a car driver in the UK with a "first epileptic seizure/solitary fit [incurs] one year off driving with medical review before restarting driving."

If I had known at the time of my initial search that SIGN (Scottish Intercollegiate Guidelines Network, www.sign. ac.uk) had produced epilepsy guidelines (April 2003) or that Clinical Evidence (www.clinicalevidence.com) covers the topic of epilepsy (March 2003), then I could have Googled rapidly to their summaries of the same meta-analysis. ${ }^{3}$

\section{ALTERNATIVE PUBMED ROUTES TO THE SAME EVIDENCE}

I noticed on my "first convulsion" search that many of the citations identified used the word "seizure" rather than "convulsion." The table above shows how some alternative search terms panned out. "Clinical Queries" is clearly a much better option than a standard PubMed search as it filtered out

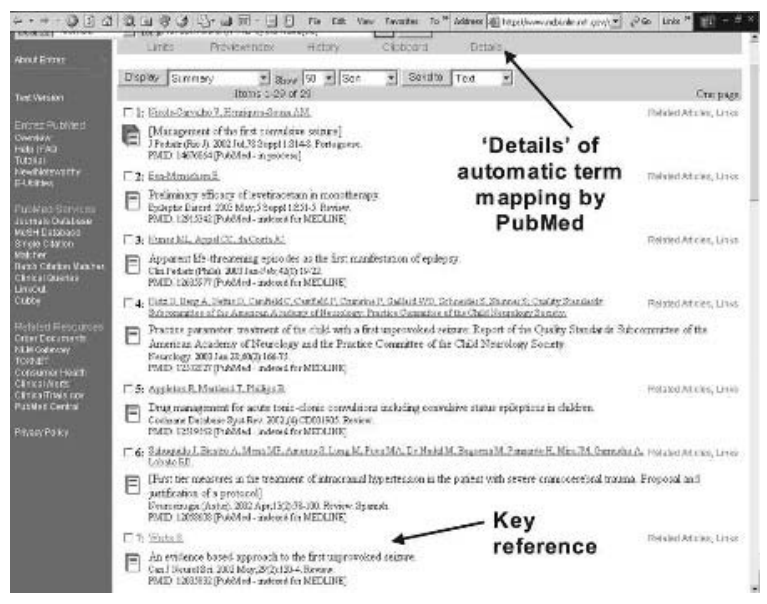

Figure 2.

more than $75 \%$ of the citations. However, both the broad and narrow clinical query searches (respectively filtering out $75 \%$ and $90 \%$ of citations) still produced far more citations than I'd time to scroll through in the clinic. The "Systematic Reviews" option consistently produced the most manageable number of citations, filtering out some $99 \%$ of the citations while still including my 2 key references. ${ }^{2}{ }^{3}$

The "systematic reviews" option pastes "systematic[sb]" into the search (sb stands for subject subset). This filter preferentially identifies systematic reviews, meta-analyses, evidence-based guidelines, and pre-appraised resources (such as the ACP Journal Club). The search involving "Seizures [MeSH]" was the most useful approach for identifying the smallest number citations, but it took slightly longer to construct (and it only filtered out an additional 3 citations compared with my original search). It involved using the "MesH Database" option in PubMed to look up the appropriate Medical Subject Heading [MesH] for my term "convulsion."

\section{AUTOMATIC TERM MAPPING}

I was puzzled that neither of the 2 key references ${ }^{2-3}$ from my "first convulsion" search actually contained the word "convulsion" anywhere in their PubMed entry. How had I managed to pick them up with my simple search? Checking "PubMed Help" revealed that unqualified searches (like "convulsion") are mapped against something called a MeSH Translation Table. This automatically matches such words against clinical synonyms, English language variants, and the relevant Medical Subject Headings (MeSH). The "details" option (figure 2) reveals what goes on behind the scenes in PubMed. My simple use of the word "convulsion: was translated into: 
70

\section{"seizures"[MeSH] OR "convulsions"[MeSH] OR convulsion[Text Word]}

With its in-built filters (and automatic term mapping), PubMed Clinical Queries is a key resource for rapid searching. It performs equally well for questions of diagnosis, therapy, and harm. ${ }^{5}$ It's particularly useful for peripatetic physicians. On any web linked PC I can rapidly access PubMed (just Google PubMed) without the need to remember any usernames or passwords.

\section{CLINICAL UNCERTAINTY}

Clinical practice is an inherently uncertain activity. Although EBM can't remove this uncertainty, it can at least help to reduce it. A recurrence risk of $40 \%$ (at 2 years) is considerably lower than my initial estimate of $70 \%$ (at 1 year). While I still can't predict the future for Susan, I can at least contribute the best currently available evidence to our discussions. I telephoned Susan and her mother to explain that the outlook was more optimistic than I had originally thought.

\section{MIKE CRILLY, MD, MPH, MRCGP, MFPHM University of Aberdeen Medical School} Aberdeen, UK

1 OPCS. Morbidity statistics from general practice. Fourth national study (MSGP4): 1991-1992. Series MB5 (no. 3). London: HMSO, 1994.

2 Wiebe S. An evidence based approach to the first unprovoked seizure. Can J Neurol Sci 2002;29:120-4.

3 Berg AT, Shinnar S. The risk of seizure recurrence following a first unprovoked seizure: a quantitative review. Neurology 1991;41:965-72.

4 Oxman AD, Cook DJ, Guyatt GH. Users' guides to the medical literature. VI. How to use an overview. Evidence-Based Medicine Working Group. JAMA 1994;272:1367-71.

5 Crilly M, Foy R. Evidence-based family planning: finding answers to clinical questions. J Fam Plann Reprod Health Care 2003;29:48-52.

\section{Weighted event rates}

W hen reporting results for dichotomous outcomes (for example, alive or dead) of systematic reviews, the event rates are labelled "weighted event rates." This weight reflects the relative contributions of individual studies to the overall risk difference, which is then used to compute the numbers needed to treat (NNTs).

The main factor in determining the weight for a trial is the number of events, which in turn depends on sample size, the

\begin{tabular}{|c|c|c|c|}
\hline \multicolumn{2}{|c|}{ Weighted event rates } & \multirow[b]{2}{*}{$\operatorname{RRR}(95 \% \mathrm{Cl})$} & \multirow[b]{2}{*}{ NNT (Cl) } \\
\hline LMWH & UFH & & \\
\hline $3.30 \%$ & $5.30 \%$ & $30 \%(11$ to 46$)$ & 50 (34 to 100$)$ \\
\hline \multicolumn{2}{|c|}{ Unweighted event rates } & & \\
\hline LMWH & UFH & & \\
\hline $3.57 \%$ & $5.30 \%$ & $33 \%(14$ to 47$)$ & 58 (36 to 148 ) \\
\hline
\end{tabular}

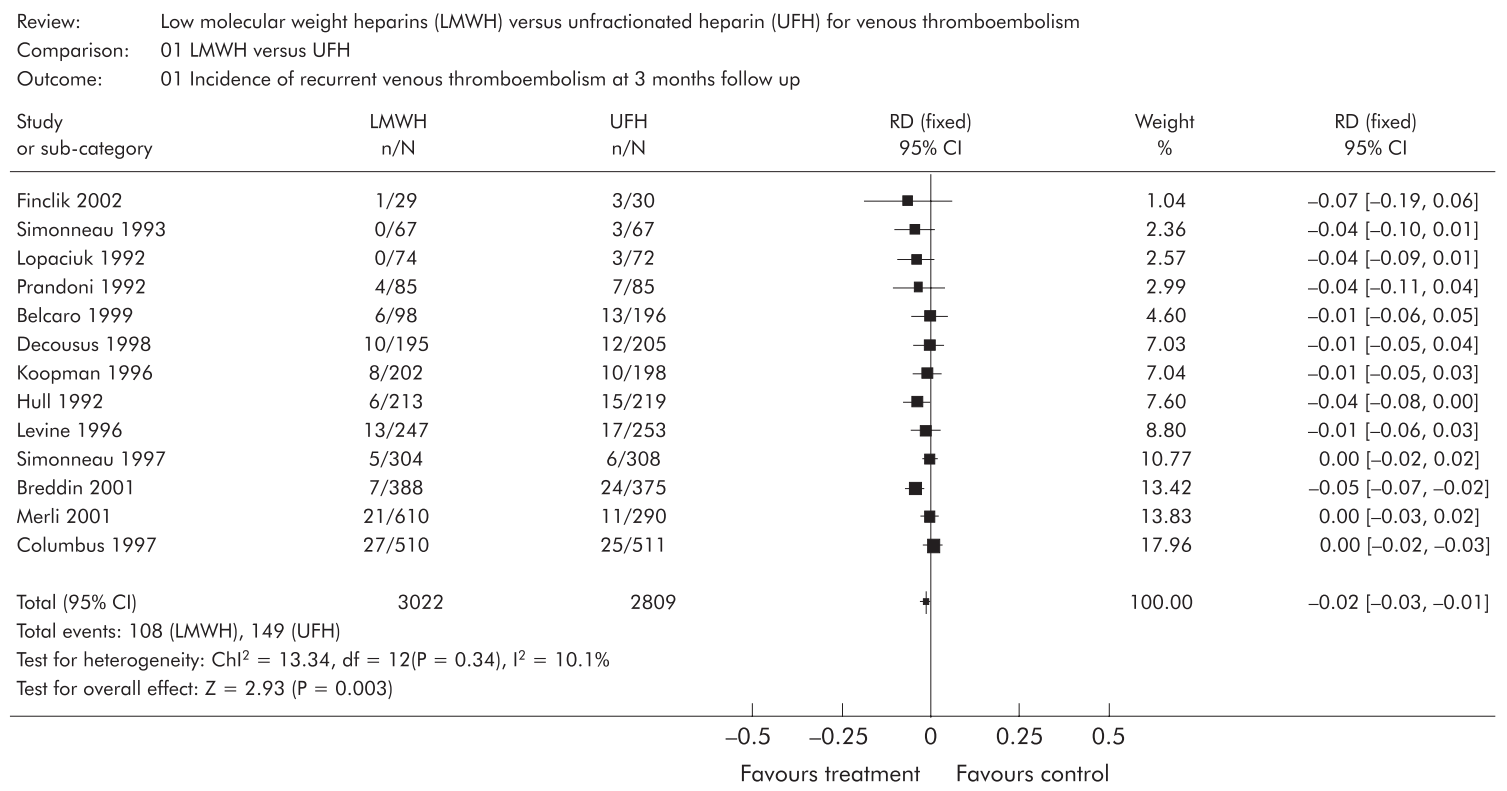

From reference 2, with permission. 
event rate, and duration of follow up. Trials with a higher number of events get more weight. The number of events and total number of patients in each of the treatment arms are combined into a single statistical concept called variance. Variances can be calculated for the event rates in each of the treatment arms, for the risk difference between the treatment groups, or for the relative risk. A good example of a weight would then be 1 /variance.

Computer software is available to do most of the computations (eg, RevMan, SAS, and Stata). For a fixed effects model, RevMan (which is probably familiar to most reviewers) will generate a Mantel Haenszel risk difference together with a corresponding weight for each of the trials, whereas the DerSimonian and Laird method is used for the random-effects model. ${ }^{1}$

A review by van Dongen et $a^{2}$ (abstracted in this issue of Evidence-Based Medicine) illustrates the principles with incidence of recurrent venous thromboembolism at 3 months as the outcome of interest (figure).

The weighted risk difference between the treatment and control groups, is obtained by adding individual trial's weight times the risk difference as follows: $(1.04 \%$ of -0.07$)+$ $(2.36 \%$ of -0.04$)+(2.57 \%$ of -0.04$)+(2.99 \%$ of -0.04$)+$ $(4.60 \%$ of -0.01$)+(7.03 \%$ of -0.01$)+(7.04 \%$ of -0.01$)+$ $(7.60 \%$ of -0.04$)+(8.80 \%$ of -0.01$)+(10.77 \%$ of 0.00$)+$ $(13.42 \%$ of -0.05$)+(13.83 \%$ of 0.00$)+(17.96 \%$ of 0.00$)=$ $-0.02(95 \% \mathrm{CI}-0.02$ to -0.01$)$. (It is noteworthy that in this case, a linear relationship exists between sample size and the weights.)

The overall event rate in the control (unfractionated heparin) group is calculated by simply pooling the results to give $149 / 2809=5.30 \%$. The overall experimental event rate is obtained by subtracting the weighted risk difference from the control event rate (ie, $5.30 \%-2.00 \%=3.30 \%$ ). Both rates are subsequently labelled "weighted event rates." (In the case of a risk increase, the weighted risk difference is added to the control event rate to get the weighted experimental event rate.) Weighted and unweighted event rates from this example are presented in the table.

The reported relative risk reductions (RRRs) from metaanalyses are similarly weighted. A consequence of this approach is that the "eyeball calculation" of the RRR-when you quickly calculate the RRR in your head from the event rates-doesn't come out to what you would expect.

However, we feel this presentation gives the most accurate report of the event rates, and the "eyeball calculation" of the NNT will come out to what you would expect.

STEPHEN R WERRE, PhD CINDY WALKER-DILKS, MLS
McMaster University, Hamilton, Ontario, Canada

1 Alderson P, Green S, Higgins JP, eds. Cochrane Reviewers' Handbook 4.2.3 (updated November 2004). www.cochrane.org/resources/handbook/ hbook.htm.

2 van Dongen CJ, van den Belt AG, Prins MH, et al. Cochrane Database Syst Rev 2004;(4):CD001 100.

\section{Letter: From research evidence to context: the challenge of individualising care}

$\mathrm{D}$ $r$ Weiner's editorial on the challenge of individualising care $^{1}$ highlights an important point that has been neglected, for the most part, in the medical literature. The enthusiasm for evidence-based medicine (the jargon is "best evidence") has obscured the fact that Weiner emphasises so well. Contextualisation, which he defines as the process of identifying factors specific to a patient's life situation and relevant to their care, is essential in the practice of internal medicine. It is what all practitioners must do to be effective and efficient.
The emphasis on quantitation above all else spills over into other disciplines; ${ }^{2}$ we must make sure that we do not neglect all aspects of our patients' lives in our single minded pursuit of statistical significance.

IRWIN J SCHATZ, MD John A. Burns School of Medicine University of Hawaii Honolulu, Hawaii, USA

1 Weiner SJ. From research evidence to context: The challenge of individualizing care. Evidence-Based Medicine 2004;9:132-3.

2 Schatz E, Schatz IJ. Medicine and political science: parallel lessons in methodological excess. PS: Political Science \& Politics 2003;36:417-22. 